\title{
Quantifying the Protection Factor of Graphene Substrates for Atomic-scale Imaging of Organic Crystals
}

\author{
Blanka Janicek, Priti Kharel, Sang hyun Bae and Pinshane Huang \\ University of Illinois at Urbana-Champaign, Urbana, Illinois, United States
}

Graphene substrates have been proposed as ultra-thin supports for dose-sensitive samples, where they increase dose resistance as a result of high thermal and electrical conductivity. For example, a single layer of graphene has provided a 9-fold protection factor when imaging single-layer $\mathrm{MoS}_{2}$ [1]. Unlike for inorganic crystals, graphene's protection factor for organic molecular crystals has yet to be systematically quantified. Here we use selected area electron diffraction (SAED) to measure the critical dose of both bulk and 2D bilayer organic crystals on either amorphous carbon (a-C) or single-layer graphene substrates. We find that the single layer of graphene offers a 2-7-fold improvement in the dose resistance. This improvement is significant: for example, increasing the critical dose by a factor of four should double the dose limited resolution for organic molecules [2]. Importantly, by comparing the improvement in critical dose for different d-spacings, we find that the protection factor provided by graphene is higher for higherorder diffraction spots. Our results indicate that graphene is especially useful for enabling near atomicresolution imaging of dose-sensitive samples, which we demonstrate by using aberration-corrected scanning transmission electron microscopy (ADF-STEM) to acquire images of cobalt(II) meso-tetrakis(4methoxyphenyl) porphyrin (CoTMPP).

CoTMPP is a metalloporphyrin, structure in Figure 2c, an important class of molecules that are essential to key biological processes, including photosynthesis, oxygen transport, and oxygen activation, and have been proposed for use in photodynamic therapy, drug delivery, and single-cell imaging [3]. Porphyrins are ideal samples for electron microscopy because they are aromatic and quasi-planar, and they have high critical doses of 60-250 $\mathrm{e}^{-} / \AA^{2}$ [4]. We compare two samples: bulk crystals dry deposited onto a-C grids and thin crystals ( $\sim 2$ molecular layers) of CoTMPP thermally evaporated onto graphene grids, fabricated as detailed in reference [5]. We next perform SAED at $300 \mathrm{kV}$ with a dose rate of $1.2-1.3 \mathrm{e}^{-} / \AA^{2}$ s. Figure 1a-c shows the normalized spot amplitude plotted as a function of applied dose for three diffraction peaks for each of the two samples. For bulk crystals the critical doses range from 11-70 $\mathrm{e}^{-} / \AA^{2}$, while for thin crystals on graphene the critical doses range from 74-156 $\mathrm{e}^{-} / \AA^{2}$. By taking the ratio of the two lines, we plot the graphene protection factor as a function of scattering vector $\mathrm{k}$. The graphene protection factors range from 2-7x and increase as a function of $\mathrm{k}$ (Figure 2a-b). These values are likely a lower bound of the real protection factor because, in the absence of graphene, we would expect that the critical dose would be lower in the 2D bilayer crystal as a result of its lower density and higher surface area [2, 4].

Next, we show that the high protection offered by graphene enables atomic-scale imaging of CoTMPP via ADF-STEM. We image bilayer CoTMPP at $300 \mathrm{kV}$ using a dose of $51 \mathrm{e}^{-} / \AA^{2}$. Notably, this dose is well above the 11-29 $\mathrm{e}^{-} / \AA^{2}$ critical doses for high-resolution information in the absence of a graphene substrate. A representative image is shown in Figure 2d, which was generated by class averaging 2000 equivalent sites in a single molecular crystal. In this image we can distinguish the four-fold symmetry of the molecule including the orientation of the methoxyphenyl groups, demonstrating graphene's utility in enabling atomic-scale imaging of CoTMPP [6]. 

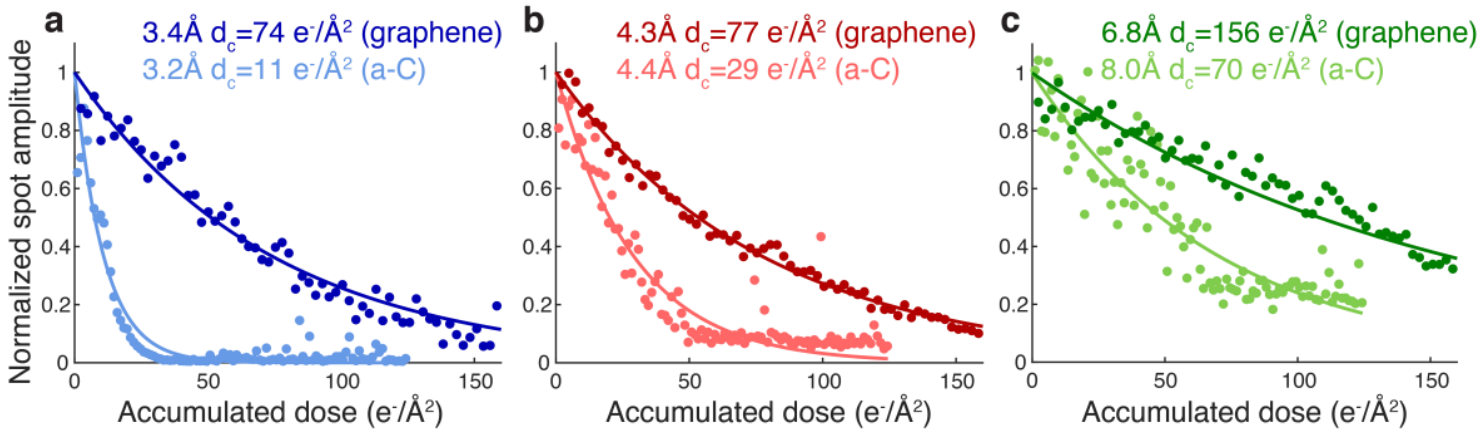

Figure 1. Effect of graphene substrates on the critical dose of CoTMPP. (a-c) Plots of the normalized diffraction spot amplitude as a function of accumulated electron dose of bulk on a-C and as-grown-on graphene crystals, acquired at $300 \mathrm{keV}$. The data (points) and exponential fit (line) are plotted for both the a-C (light color) and graphene (dark color) crystals. Because the crystal structures and zone axes differ between the two samples, we compare the most similar d-spacings, approximately $3 \AA$ (a), $4 \AA$ (b), and 7 $\AA$ (c). For all plots we see that graphene significantly increases the critical dose, offering a 2-7-fold improvement.
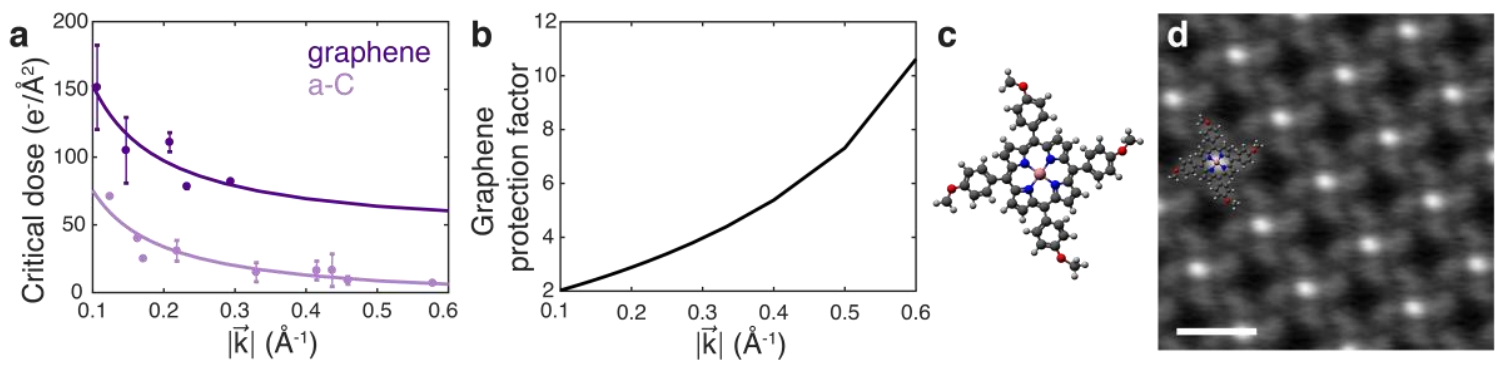

Figure 2. Trends in critical dose with scattering vector and class averaged ADF-STEM image of bilayer CoTMPP on graphene. (a) Plot of the critical dose as a function of scattering vector. All the data (points) and are shown for a-C (light purple) and graphene (dark purple) crystals with a linear fit to the mean. (b) Graphene protection factor versus scattering vector k. Graphene's protection factor increases for larger k. (c) Structure of CoTMPP. (d) Class averaged ADF-STEM image (2000 frames) of bilayer CoTMPP crystal on graphene, with cartoon structure overlaid. The image was acquired with 51 e-/Å2. The scale bar is $10 \AA$.

\section{References}

[1] G Algara-Siller et al., Applied Physics Letters 12 (2013), p. 203107.

[2] R Egerton, Micron 119 (2019), p. 72.

[3] M Imran, et al., Biosensors 8 (2018), p. 95.

[4] L Reimer and H Khol in "Transmission Electron Microscopy: Physics of Image Formation”, ed. W Rhodes, (Springer, ) p.474.

[5] B Janicek et al., Nano Letters 19 (2019), p. 6308.

[6] This work was supported by the Packard Foundation and an NSF CAREER award under DMR1846206. The experimental work was carried out in the Materials Research Laboratory. 